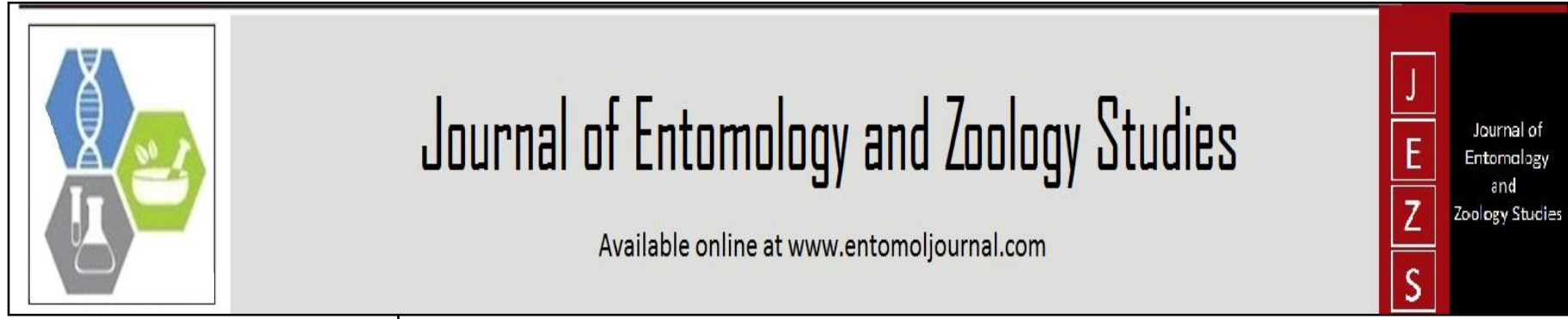

E-ISSN: 2320-7078

P-ISSN: 2349-6800

www.entomoljournal.com JEZS 2020; 8(1): 1592-1596 (C) $2020 \mathrm{JEZS}$

Received: 09-11-2019

Accepted: 13-12-2019

\section{Subhajit Pal}

Department of Agricultural

Entomology, Institute of

Agriculture, Visva-Bharati,

Sriniketan, West Bengal, India

Swarnali Bhattacharya

Department of Agricultural

Entomology, Institute of

Agriculture, Visva-Bharati,

Sriniketan, West Bengal, India

Shrawan Kumar Sahani

Department of Agricultural

Entomology, Institute of

Agriculture, Visva-Bharati,

Sriniketan, West Bengal, India
Corresponding Author: Subhajit Pal

Department of Agricultural

Entomology, Institute of

Agriculture, Visva-Bharati,

Sriniketan, West Bengal, India

\section{Population dynamics of whitefly (Bemisia tabaci Genn.) infesting BT cotton and their insecticidal management under field conditions}

\author{
Subhajit Pal, Swarnali Bhattacharya and Shrawan Kumar Sahani
}

DOI: https://doi.org/10.22271/j.ento.2020.v8.i1aa.6372

\section{Abstract}

Studies were taken up to monitor the seasonal occurrence of whitefly population in cotton as well as its insecticidal management under field conditions of Institutional Research Farm, Institute of Agriculture, Visva-Bharati, Sriniketan, during the kharif season of 2018-19. Population reduction of whitefly was done with eight insecticides having different molecular composition and one untreated check. The experiment was drawn in a Randomised Block Design with three replications. The occurrence of whitefly was noticed during the fourth week of July ( 0.2 whitefly per plant) which continued till late December. Highest whitefly population was found during fourth week of September (12.24 Whitefly per plant). Correlation studies of whitefly population and abiotic factors suggested that whitefly population was significantly correlated with maximum temperature at $1 \%$ level of significance. Among the different insecticides tested for population reduction of whitefly in Bt cotton it was noticed that combination product of Imidacloprid 6\% + Lambda Cyhalothrin 4\%SL @ $500 \mathrm{ml} / \mathrm{ha}$ and $450 \mathrm{ml} / \mathrm{ha}$ proved most effective and gave $94.15 \%$ and $93.81 \%$ pest population reduction after second spray imposition respectively.

Keywords: Whitefly, insecticide, seasonal occurrence, maximum temperature

\section{Introduction}

Cotton (Gossypium spp.) is one of the most vital revenue generating crop in our country playing a greater importance in agricultural, industrial, socio economical and pecuniary affairs of the country ${ }^{[13]}$. Globally India takes the first position in area (about $35 \%$ of the world) and second in production, next to china [7]. As per Cotton Advisory Board expected cotton production in India during 2017-18 is 377 lakh bales of $170 \mathrm{~kg}$ from 122 lakh hectares with a productivity of $524 \mathrm{~kg}$ lint/ha ${ }^{[8]}$. Among the various reasons which relate to the check points in cotton production, biotic stresses accounts to a major factor. Till date, 1326 species of insects and mites have been recorded worldwide ${ }^{[6]}$. But with the commercial cultivation of Btcotton crop there was not only change in the cultivation profile, but also the pest scenario. BT cotton provided effective management of bollworm complex but sucking insect pests namely whitefly (Bemisia tabaci), leafhopper (Amrasca biguttula), aphid (Aphis gossypii), mealybug (Phenacoccus solenopsis) and thrips (Thrips tabaci) turned out be emerging as serious pests $[11,12,19]$.

Among the sucking pests, whitefly is one of the notorious and key pest due to its polyphagous nature and ability to adapt to diverse cropping system ${ }^{[4,18] . ~ C o n s t a n t l y ~ s u c k i n g ~ t h e ~ c e l l ~ s a p ~}$ results in $50 \%$ reduction in boll production hindering the photosynthesis by secreting honey dew on which sooty mould develops [2]. Acts as a major vector of leaf curl virus disease causing average yield loss of $38.7 \%{ }^{[10,16]}$. Several pest control techniques are utilized to manage the dreaded pest but the most common and quicker one is the chemical control which is generally adopted by our farming community. Therefore the objective of the study was coined to have a proper knowledge on the seasonal incidence, population dynamics and insecticidal management of whitefly infesting cotton because without proper knowledge on seasonal incidence of the pest and proper selection of insecticide desirable management cannot be achieved. 


\section{Materials and Methods}

The present study was conducted at the Institutional Research Farm, Institute of Agriculture, Visva-Bharati, Sriniketan, during kharif season of 2018-19. Bt cotton cultivar KCH-149 (BGII) was cultivated following all the recommended agronomic practices and intercultural operations for well establishment of the crop. The crop was sown on June 15, 2018 in an isolated plot of 432 sq. meter comprising of $4 \mathrm{~m} \mathrm{X}$ $4 \mathrm{~m}$ each plot size by giving a recommended spacing of $90 \mathrm{~cm}$ X $60 \mathrm{~cm}$. The nos. of whitefly were recorded from 50 randomly selected plants from three leaves (top, middle and bottom) and later average was calculated. The data was recorded at weekly interval starting from twenty days after sowing till the crop was harvested. With regards to the experiment with insecticidal treatment Randomised Block Design was taken with three replications and nine treatments which included eight treatments with insecticides and an untreated control. One pre-treatment data on whitefly and data on $1^{\text {st }}, 3^{\text {rd }}, 5^{\text {th }}, 7^{\text {th }}$ and $14^{\text {th }}$ days after treatment imposition was taken. The data was transformed accordingly and statistical calculation was done to find out the significant treatment. The weekly weather parameters, viz. maximum temperature, minimum temperature, relative humidity, temperature gradient, rainfall and sunshine hour were obtained from Meteorological Office Sriniketan, Birbhum, and WB. For statistical analysis SPSS version 16 has been used to find out possible relationship of whitefly population with various meteorological factors.

\section{Results and Discussions \\ Seasonal incidence of whitefly infesting cotton}

Whitefly was found to infest cotton for the first time in the fourth week of July where about 0.2 whitefly population per plant was observed. Highest whitefly population was found during the fourth week of September (12.24 Whitefly per plant) with the maximum temperature $35.3{ }^{\circ} \mathrm{C}$, minimum temperature $25.61{ }^{\circ} \mathrm{C}$, relative humidity $81 \%$, rainfall at the rate of $1.06 \mathrm{~mm}$ and sunshine hours 6.76 . The population of whitefly continued to stay on cotton from the fourth week of July to late December i.e. vegetative stage of the plant to maturity stage. Similar results have been found out by several workers where incidence of whitefly started from the 5th WAS i.e. the second week of August (0.38 whitefly/leaf). The pest population increased gradually up to the $14^{\text {th }}$ WAS and thereafter, increased rapidly and showed peak activity at the $18^{\text {th }}$ WAS i.e. the second week of November $(12.60$ whiteflies/leaf) ${ }^{[5]}$. In 2011, activity of whitefly was noticed throughout the cotton growth period ${ }^{[3]}$.

Table 1: Seasonal incidence of whitefly infesting cotton with respect to certain abiotic parameters during the year 2018-19.

\begin{tabular}{|c|c|c|c|c|c|c|c|c|}
\hline \multirow[b]{2}{*}{$\begin{array}{c}\text { Standard } \\
\text { Week }\end{array}$} & \multirow{2}{*}{$\begin{array}{c}\text { Whitefly } \\
\text { population / } \\
\text { plants }\end{array}$} & \multicolumn{7}{|c|}{ Important weather parameters as recorded during the respective standard week } \\
\hline & & \begin{tabular}{|c|} 
Correlated with \\
the standard week
\end{tabular} & $\begin{array}{c}\text { Maximum } \\
\text { Temperature }\left({ }^{0} \mathrm{C}\right)\end{array}$ & \begin{tabular}{|c|} 
Minimum \\
Temperature $\left({ }^{0} \mathrm{C}\right)$
\end{tabular} & $\begin{array}{l}\text { Temperature } \\
\text { Gradient }\left({ }^{0} \mathrm{C}\right)\end{array}$ & $\begin{array}{c}\text { Relative } \\
\text { Humidity (\%) }\end{array}$ & $\begin{array}{c}\text { Rainfall } \\
\text { (mm) }\end{array}$ & $\begin{array}{l}\text { Sunshine } \\
\text { Hours }\end{array}$ \\
\hline $1^{\text {st }}$ & 0.20 & $30^{\text {th }}$ & 31.49 & 26.30 & 5.19 & 88.29 & 18.21 & 1.06 \\
\hline $2^{\text {nd }}$ & 0.13 & $31^{\text {th }}$ & 32.66 & 26.01 & 6.65 & 83.86 & 1.73 & 1.96 \\
\hline $3^{\text {rd }}$ & 0.47 & $32^{\text {th }}$ & 33.74 & 26.66 & 7.08 & 84.29 & 5.03 & 6.06 \\
\hline $4^{\text {th }}$ & 1.38 & $33^{\text {th }}$ & 34.54 & 27.21 & 7.33 & 81.71 & 0.94 & 6.97 \\
\hline $5^{\text {th }}$ & 1.93 & $34^{\text {th }}$ & 33.80 & 26.70 & 7.10 & 82.86 & 7.32 & 5.20 \\
\hline $6^{\text {th }}$ & 4.42 & $35^{\text {th }}$ & 32.80 & 26.39 & 6.41 & 82.57 & 7.44 & 5.33 \\
\hline $7^{\text {th }}$ & 6.53 & $36^{\text {th }}$ & 33.14 & 25.73 & 7.41 & 84.57 & 9.94 & 6.19 \\
\hline $8^{\text {th }}$ & 8.20 & $37^{\text {th }}$ & 34.13 & 25.77 & 8.36 & 83.86 & 11.83 & 5.40 \\
\hline $9^{\text {th }}$ & 10.20 & $38^{\text {th }}$ & 33.21 & 25.20 & 8.01 & 81.86 & 6.57 & 5.96 \\
\hline $10^{\text {th }}$ & 12.24 & $39^{\text {th }}$ & 35.30 & 25.61 & 9.69 & 81.00 & 1.06 & 6.76 \\
\hline $11^{\text {th }}$ & 5.43 & $40^{\text {th }}$ & 35.07 & 23.39 & 11.68 & 73.71 & 0.00 & 3.26 \\
\hline $12^{\text {th }}$ & 6.20 & $41^{\text {th }}$ & 30.27 & 22.56 & 7.71 & 82.57 & 5.11 & 2.46 \\
\hline $13^{\text {th }}$ & 6.00 & $42^{\text {th }}$ & 33.34 & 20.50 & 12.84 & 79.29 & 0.00 & 2.64 \\
\hline $14^{\text {th }}$ & 5.98 & $43^{\text {th }}$ & 2.19 & 18.84 & 13.35 & 74.71 & 0.31 & 2.50 \\
\hline $15^{\text {th }}$ & 6.00 & $44^{\text {th }}$ & 31.44 & 20.49 & 10.95 & 79.43 & 0.00 & 5.96 \\
\hline $16^{\text {th }}$ & 6.42 & $45^{\text {th }}$ & 31.11 & 17.86 & 13.25 & 75.57 & 0.00 & 7.14 \\
\hline $17^{\text {th }}$ & 7.00 & $46^{\text {th }}$ & 31.27 & 15.97 & 15.30 & 77.29 & 0.00 & 7.26 \\
\hline $18^{\text {th }}$ & 5.24 & $47^{\text {th }}$ & 30.01 & 12.86 & 17.15 & 75.14 & 0.00 & 7.84 \\
\hline $19^{\text {th }}$ & 3.46 & $48^{\text {th }}$ & 29.46 & 15.86 & 13.60 & 74.86 & 0.00 & 6.10 \\
\hline $20^{\text {th }}$ & 4.42 & $49^{\text {th }}$ & 27.53 & 12.03 & 15.55 & 78.00 & 0.00 & 6.69 \\
\hline $21^{\text {th }}$ & 0.72 & $50^{\text {th }}$ & 28.14 & 12.73 & 15.41 & 71.86 & 0.00 & 6.33 \\
\hline $22^{\text {th }}$ & 0.56 & $51^{\text {th }}$ & 22.54 & 11.96 & 10.58 & 87.29 & 4.07 & 5.26 \\
\hline $23^{\text {th }}$ & 0.12 & $52^{\text {th }}$ & 24.34 & 8.15 & 16.19 & 72.38 & 0.00 & 8.50 \\
\hline
\end{tabular}

Ecological parameters played a major factor on the population fluctuation of whitefly shown in Fig-1. The variation of Whitefly population among the different standard weeks of observations showed highest significant Whitefly populations in $10^{\text {th }}$ standard weeks that is 12.24 Whitefly per plant. On the other hand $9^{\text {th }}, 8^{\text {th }}, 17^{\text {th }}, 7^{\text {th }}, 16^{\text {th }}, 12^{\text {th }}, 13^{\text {th }}, 15^{\text {th }}$ and $14^{\text {th }}$ standard week population were at par with each other, whereas $18^{\text {th }}$, $19^{\text {th }}, 20^{\text {th }}, 22^{\text {th }}, 23^{\text {th }}, 11^{\text {th }}, 6^{\text {th }}, 5^{\text {th }}, 4^{\text {th }}, 3^{\text {rd }}, 2^{\text {nd }}$ and $1^{\text {st }}$ standard week's Whitefly population was at par with each other. 


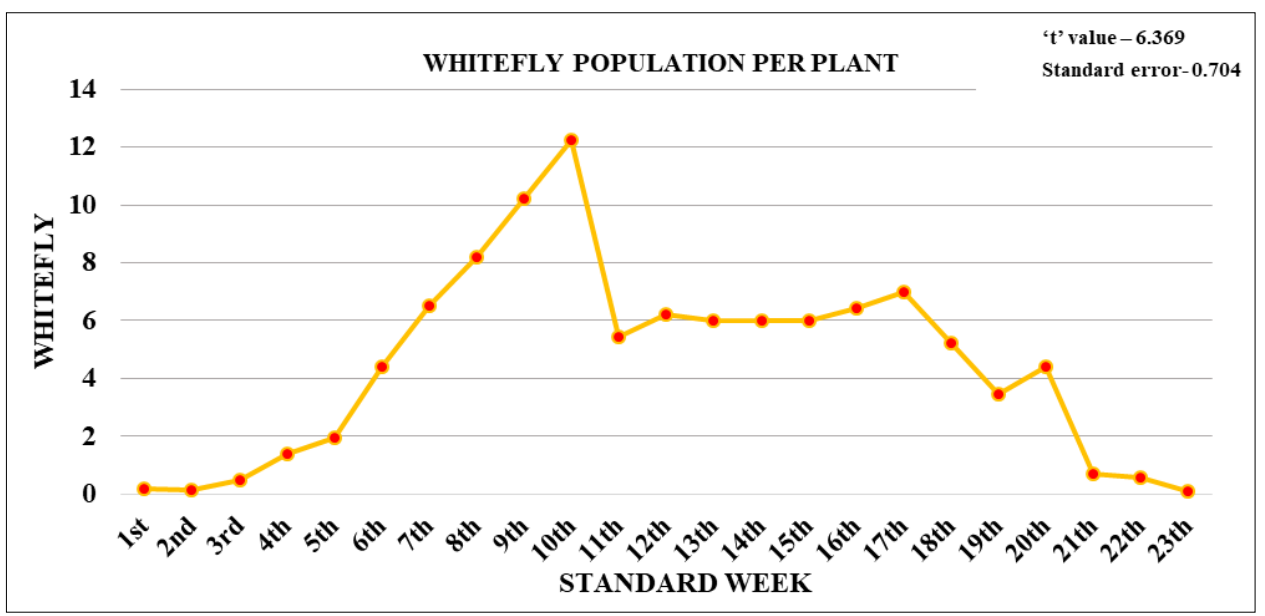

Fig 1: Population variation of Whitefly population in different standard week

Correlation studies of weather parameters with whitefly population. Multivariate correlation of whitefly population with weather parameters

The multivariate correlation studies (Table-2) suggests that maximum temperature is significantly positively correlated with the Whitefly population at $1 \%$ level of significance. The other weather parameters like minimum temperature, temperature gradient, relative humidity, rainfall and sunshine hours were not related with the population fluctuation of
Whitefly.

In $\mathrm{Bt}$ cotton genotypes, the maximum temperature was significantly and positively correlated with whitefly population while negatively correlated with rainfall [17,9] which is in close conformity with the present findings. Whitefly population showed significantly negative correlation with minimum temperature and relative humidity at morning and evening ${ }^{[14,17]}$.

Table 2: Multivariate correlation between different weather parameters and whitefly population

\begin{tabular}{|c|c|c|c|c|c|c|c|}
\hline Parameter & Whitefly & Max. Temp. & Min. Temp. & Temp. Grad. & RH & Rainfall & SH \\
\hline Whitefly & 1 & & & & & & \\
\hline Max. Temp. & $.442^{*}$ & 1 & & & & & \\
\hline Min. Temp. & .199 & $.849^{* *}$ & 1 & & & & \\
\hline Temp. Grad. & .063 & $-.508^{*}$ & $-.886^{* *}$ & 1 & & & \\
\hline RH & -.087 & .199 & $.622^{* *}$ & $-.840^{* *}$ & 1 & & \\
\hline Rainfall & -.069 & .210 & $.551^{* *}$ & $-.715^{* *}$ & $.747^{* *}$ & 1 & \\
\hline SH & .131 & -.258 & $-.425^{*}$ & $.467^{*}$ & -.373 & -.372 & 1 \\
\hline
\end{tabular}

$1 \%$ level of significance $(* *)$

$5 \%$ level of significance $(*)$

\section{Evaluation of some newer insecticide molecule against whitefly infestation on bt-cotton}

Evaluation of insecticide molecules was carried out with nine different treatments including one untreated control depicted in Table 3 and Table 4. The results of the experiment reveals that the first and second spray gave similar results in population reduction of whitefly. For first spray the pretreatment data ranged from 9.55 to 10.15 where the second spray it was 1.32 to 12.87 . From the first spray it is evident that the treatment with Imidacloprid $6 \%+$ Lambda Cyhalothrin 4\% SL (INOVEXIA) @ $500 \mathrm{ml} / \mathrm{ha}$ gave the best results recording $73.24 \%$ population reduction of Whitefly followed by Imidacloprid 6\% + Lambda Cyhalothrin 4\% SL (INOVEXIA) @ $450 \mathrm{ml} / \mathrm{ha}$ and Imidacloprid 6\% + Lambda Cyhalothrin 4\% SL (INOVEXIA) @ $400 \mathrm{ml} / \mathrm{ha}$ recording $71.01 \%$ population reduction. On the other hand, the treatments with Buprofezin 25\% SC @ $1000 \mathrm{ml} / \mathrm{ha}$, Lambda Cyhalothrin 5\% EC @ 500 ml/ha, Diafenthuron 50\% WP @ $600 \mathrm{~g} / \mathrm{ha}$, Imidacloprid 6\% + Lambda Cyhalothrin 4\% SL (INOVEXIA) @ $350 \mathrm{ml} / \mathrm{ha}$ and Imidacloprid 70\% WG @ 35 $\mathrm{g} / \mathrm{ha}$ recorded the population reduction of whitefly to the tune of $62.52,61.93,58.49,49.74$ and 47.31 percent respectively. Here it can be mentioned that the treatment with Buprofezin 25\% SC and Lambda Cyhalothrin 5\% EC gave quite similar result. Similar results were obtained in the second spray also where Imidacloprid 6\% + Lambda Cyhalothrin 4\%SL @ 500 $\mathrm{ml} / \mathrm{ha}$ gave the best results with $94.15 \%$ of whiteflies population reduction. The next best treatments were in the order of Imidacloprid 6\% + Lambda Cyhalothrin 4\%SL @ $450 \mathrm{ml} / \mathrm{ha}>$ Imidacloprid 6\% + Lambda Cyhalothrin 4\%SL @ $400 \mathrm{ml} / \mathrm{ha}>$ Lambda Cyhalothrin 5\% EC @ $500 \mathrm{ml} / \mathrm{ha}>$ Buprofezin 25\% SC @ $1000 \mathrm{ml} / \mathrm{ha}>$ Diafenthuron 50\% WP $600 \mathrm{~g} / \mathrm{ha}>$ Imidacloprid 6\% + Lambda Cyhalothrin 4\% SL @ $350 \mathrm{ml} / \mathrm{ha}$. Similar reports of maximum population reduction of whitefly to the tune of $91 \%$ was recorded with Imidachloprid $25 \% \mathrm{WP}$ at 1 DAS ${ }^{[1]}$. Likewise, other scientist reported that the maximum percent reduction in whitefly population with Difenthiuron $50 \mathrm{WP}$ and it was statistically at par with flonicamid 50 WP @ 100g a.i. and studies proved that acetamiprid 20 SP @ 0.004 per cent was effective in lowering down the whitefly population ( 0.99 whiteflies/leaf) [15]. 
Table 3: Effect of different insecticides against Whitefly infesting in Bt Cotton (2018)

\begin{tabular}{|c|c|c|c|c|c|c|c|c|c|c|}
\hline \multirow[t]{2}{*}{ S. No. } & \multirow[t]{2}{*}{ Treatment } & \multirow{2}{*}{$\begin{array}{c}\text { Dose } \\
(\mathbf{m l} / \mathbf{g} / \mathbf{h a} .)\end{array}$} & \multicolumn{6}{|c|}{$\begin{array}{l}\text { Effect of diferente insecticidas against whitefly } \\
\text { (After } 1^{\text {st }} \text { spray) }\end{array}$} & \multirow{2}{*}{\begin{tabular}{|c|} 
Mean \\
populación \\
reducción
\end{tabular}} & \multirow{2}{*}{$\begin{array}{l}\text { Percent populación } \\
\text { reduction (Abott's } \\
\text { Correction) }\end{array}$} \\
\hline & & & PT & 1 DAS & 3 DAS & 5 DAS & 7 DAS & 14 DAS & & \\
\hline 1. & \begin{tabular}{|c|} 
T1 : Imidacloprid 6\% + Lambda \\
Cyhalothrin 4\%SL (INOVEXIA)
\end{tabular} & $350 \mathrm{ml} / \mathrm{ha}$ & $\begin{array}{c}9.76 \\
(3.20)\end{array}$ & $\begin{array}{c}7.86 \\
(2.89)\end{array}$ & $\begin{array}{l}6.28 \\
(2.6)\end{array}$ & \begin{tabular}{|c|}
5.70 \\
$(2.49)$
\end{tabular} & $\begin{array}{c}5.20 \\
(2.39)\end{array}$ & $\begin{array}{c}4.45 \\
(2.22)\end{array}$ & 5.90 & 49.74 \\
\hline 2. & \begin{tabular}{|l|} 
T2 : Imidacloprid 6\% + Lambda \\
Cyhalothrin 4\%SL (INOVEXIA)
\end{tabular} & $400 \mathrm{ml} / \mathrm{ha}$ & $\begin{array}{c}9.78 \\
(3.21)\end{array}$ & $\begin{array}{c}6.84 \\
(2.71)\end{array}$ & $\begin{array}{c}4.66 \\
(2.27)\end{array}$ & $\begin{array}{c}3.83 \\
(2.08) \\
\end{array}$ & $\begin{array}{c}2.75 \\
(1.80)\end{array}$ & $\begin{array}{c}1.57 \\
(1.44)\end{array}$ & 3.93 & 66.51 \\
\hline 3. & $\begin{array}{l}\text { T3 : Imidacloprid 6\% + Lambda } \\
\text { Cyhalothrin 4\%SL (INOVEXIA) }\end{array}$ & $450 \mathrm{ml} / \mathrm{ha}$ & $\begin{array}{c}9.55 \\
(3.17)\end{array}$ & $\begin{array}{c}5.8 \\
(2.51)\end{array}$ & $\begin{array}{c}4.11 \\
(2.15)\end{array}$ & \begin{tabular}{|c|}
3.23 \\
$(1.93$ \\
\end{tabular} & $\begin{array}{c}2.35 \\
(1.69) \\
\end{array}$ & $\begin{array}{c}1.52 \\
(1.42) \\
\end{array}$ & 3.40 & 71.01 \\
\hline 4. & $\begin{array}{l}\text { T4 : Imidacloprid 6\% + Lambda } \\
\text { Cyhalothrin 4\%SL (INOVEXIA) }\end{array}$ & $500 \mathrm{ml} / \mathrm{ha}$ & $\begin{array}{c}9.86 \\
(3.22)\end{array}$ & $\begin{array}{c}5.00 \\
(2.34)\end{array}$ & $\begin{array}{c}3.98 \\
(2.11)\end{array}$ & \begin{tabular}{|c|}
3.17 \\
$(1.91)$ \\
\end{tabular} & $\begin{array}{c}2.23 \\
(1.65)\end{array}$ & $\begin{array}{c}1.32 \\
(1.34)\end{array}$ & 3.14 & 73.24 \\
\hline 5. & T5: Lambda cyhalothrin 5\% EC & $500 \mathrm{ml} / \mathrm{ha}$ & $\begin{array}{l}10.09 \\
(3.25)\end{array}$ & $\begin{array}{c}6.45 \\
(2.64)\end{array}$ & $\begin{array}{l}4.85 \\
(2.31\end{array}$ & \begin{tabular}{|c|}
4.16 \\
$(2.16)$ \\
\end{tabular} & $\begin{array}{c}3.72 \\
(2.05)\end{array}$ & $\begin{array}{c}3.16 \\
(1.91) \\
\end{array}$ & 4.47 & 61.93 \\
\hline 6. & T6: Imidacloprid 70\% WG & $35 \mathrm{~g} / \mathrm{ha}$ & $\begin{array}{c}9.85 \\
(3.22)\end{array}$ & $\begin{array}{c}7.72 \\
(2.87)\end{array}$ & $\begin{array}{c}6.6 \\
(2.66)\end{array}$ & \begin{tabular}{|c|}
6.04 \\
$(2.56)$ \\
\end{tabular} & $\begin{array}{c}5.52 \\
(2.45)\end{array}$ & $\begin{array}{c}5.04 \\
(2.35) \\
\end{array}$ & 6.18 & 47.31 \\
\hline 7. & T7: Buprofezin 25\% SC & $1000 \mathrm{ml} / \mathrm{ha}$ & $\begin{array}{l}10.15 \\
(3.26) \\
\end{array}$ & $\begin{array}{c}6.41 \\
(2.63) \\
\end{array}$ & $\begin{array}{l}5.35 \\
(2.42 \\
\end{array}$ & \begin{tabular}{|c|}
3.99 \\
$(2.12)$ \\
\end{tabular} & $\begin{array}{c}3.42 \\
(1.98)\end{array}$ & $\begin{array}{c}2.78 \\
(1.81) \\
\end{array}$ & 4.39 & 62.59 \\
\hline 8. & T8: Diafenthuron 50\% WP & $600 \mathrm{~g} / \mathrm{ha}$ & $\begin{array}{c}9.85 \\
(3.22)\end{array}$ & $\begin{array}{c}6.27 \\
(2.60)\end{array}$ & $\begin{array}{c}5.52 \\
(2.45)\end{array}$ & \begin{tabular}{|c|}
5.12 \\
$(2.37)$
\end{tabular} & $\begin{array}{c}4.16 \\
(2.16)\end{array}$ & $\begin{array}{c}3.29 \\
(1.95)\end{array}$ & 4.87 & 58.49 \\
\hline 9. & T9: Untreated Check & - & $\begin{array}{l}10.12 \\
(3.26)\end{array}$ & $\begin{array}{l}10.35 \\
(3.29)\end{array}$ & $\begin{array}{l}11.45 \\
(3.45)\end{array}$ & $\begin{array}{l}11.93 \\
(3.52) \\
\end{array}$ & $\begin{array}{l}12.08 \\
(3.54)\end{array}$ & $\begin{array}{l}12.87 \\
(3.64)\end{array}$ & 11.74 & - \\
\hline \multirow{2}{*}{\multicolumn{3}{|c|}{$\frac{\text { C.D. }(0.05 \%)}{\operatorname{Se}(\mathrm{m})}$}} & NS & 0.67 & 0.58 & 1.08 & 0.57 & 0.65 & \multirow{2}{*}{\multicolumn{2}{|c|}{-}} \\
\hline & & & - & 0.30 & 0.27 & 0.52 & 0.14 & 0.29 & & \\
\hline
\end{tabular}

Figure in brackets represents the square root transformed value * Mean of ten plants

Table 4: Effect of different insecticides against whitefly infesting Cotton (2018)

\begin{tabular}{|c|c|c|c|c|c|c|c|c|c|c|}
\hline \multirow[t]{2}{*}{ S. $\mathbf{N}$. } & \multirow[t]{2}{*}{ Treatment } & \multirow{2}{*}{$\begin{array}{c}\text { Dose } \\
(\mathbf{m l} / \mathbf{g} / \mathbf{h a} .)\end{array}$} & \multicolumn{6}{|c|}{$\begin{array}{c}\text { Effect of different insecticides against } \\
\text { whitefly (After } 2^{\text {nd }} \text { spray) }\end{array}$} & \multirow{2}{*}{$\begin{array}{c}\text { Mean } \\
\text { population } \\
\text { reduction }\end{array}$} & \multirow{2}{*}{$\begin{array}{l}\text { Per cent population } \\
\text { reduction (Abott's } \\
\text { Correction) }\end{array}$} \\
\hline & & & PT & 1 DAS & 3DAS & 5DAS & 7DAS & 14DAS & & \\
\hline 1. & $\begin{array}{l}\text { T1 : Imidacloprid 6\% + Lambda } \\
\text { Cyhalothrin 4\%SL (INOVEXIA) }\end{array}$ & $350 \mathrm{ml} / \mathrm{ha}$ & \begin{tabular}{|c|}
4.45 \\
$(2.22)$ \\
\end{tabular} & $4.23(2.17)$ & \begin{tabular}{|c|}
3.98 \\
$(2.11)$ \\
\end{tabular} & $\begin{array}{c}3.65 \\
(2.03) \\
\end{array}$ & $\begin{array}{c}3.32 \\
(1.95) \\
\end{array}$ & $\begin{array}{c}3.00 \\
(1.87)\end{array}$ & 6.36 & 75.02 \\
\hline 2. & $\begin{array}{l}\text { T2 : Imidacloprid 6\% + Lambda } \\
\text { Cyhalothrin 4\%SL (INOVEXIA) }\end{array}$ & $400 \mathrm{ml} / \mathrm{ha}$ & \begin{tabular}{|c|}
1.57 \\
$(1.44)$
\end{tabular} & $1.25(1.32)$ & $\begin{array}{c}1.05 \\
(1.24)\end{array}$ & $\begin{array}{c}0.98 \\
(1.21)\end{array}$ & $\begin{array}{c}0.78 \\
(1.13)\end{array}$ & $\begin{array}{c}0.70 \\
(1.09)\end{array}$ & 0.95 & 93.46 \\
\hline 3. & $\begin{array}{l}\text { T3 : Imidacloprid 6\% + Lambda } \\
\text { Cyhalothrin 4\%SL (INOVEXIA) }\end{array}$ & $450 \mathrm{ml} / \mathrm{ha}$ & $\begin{array}{c}1.52 \\
(1.42) \\
\end{array}$ & $1.21(1.30)$ & $\begin{array}{r}1.00 \\
(1.22) \\
\end{array}$ & $\begin{array}{c}0.89 \\
(1.17)\end{array}$ & $\begin{array}{c}0.75 \\
(1.11) \\
\end{array}$ & $\begin{array}{c}0.67 \\
(1.08)\end{array}$ & 0.90 & 93.81 \\
\hline 4. & $\begin{array}{l}\text { T4 : Imidacloprid 6\% + Lambda } \\
\text { Cyhalothrin 4\%SL (INOVEXIA) }\end{array}$ & $500 \mathrm{ml} / \mathrm{ha}$ & $\begin{array}{c}1.32 \\
(1.34) \\
\end{array}$ & $1.12(1.27)$ & $\begin{array}{c}0.98 \\
(1.21) \\
\end{array}$ & $\begin{array}{c}0.85 \\
(1.16) \\
\end{array}$ & $\begin{array}{c}0.72 \\
(1.10) \\
\end{array}$ & $\begin{array}{c}0.60 \\
(1.04)\end{array}$ & 0.85 & 94.15 \\
\hline 5. & T5: Lambda cyhalothrin 5\% EC & $500 \mathrm{ml} / \mathrm{ha}$ & \begin{tabular}{|c|}
3.16 \\
$(1.91)$ \\
\end{tabular} & $2.28(1.67)$ & $\begin{array}{c}1.87 \\
(1.54) \\
\end{array}$ & $\begin{array}{c}1.68 \\
(1.48)\end{array}$ & \begin{tabular}{c|c|}
1.43 \\
$(1.39$
\end{tabular} & $\begin{array}{c}1.00 \\
(1.22)\end{array}$ & 1.65 & 88.64 \\
\hline 6. & T6: Imidacloprid 70\% WG & $35 \mathrm{~g} / \mathrm{ha}$ & $\begin{array}{c}5.04 \\
(2.35)\end{array}$ & $4.17(2.16)$ & $\begin{array}{c}3.70 \\
(2.05)\end{array}$ & $\begin{array}{c}3.32 \\
(1.95)\end{array}$ & \begin{tabular}{|c|}
2.78 \\
$(1.81)$ \\
\end{tabular} & $\begin{array}{c}2.32 \\
(1.68)\end{array}$ & 3.26 & 77.56 \\
\hline 7. & T7: Buprofezin 25\% SC & $\begin{array}{l}1000 \\
\mathrm{ml} / \mathrm{ha}\end{array}$ & \begin{tabular}{|c|}
2.78 \\
$(1.81)$ \\
\end{tabular} & $2.69(1.80)$ & \begin{tabular}{|c|}
2.12 \\
$(1.61)$ \\
\end{tabular} & $\begin{array}{c}2.05 \\
(1.59) \\
\end{array}$ & $\begin{array}{c}1.94 \\
(1.55) \\
\end{array}$ & $\begin{array}{c}1.64 \\
(1.42) \\
\end{array}$ & 2.08 & 85.68 \\
\hline 8. & T8: Diafenthuron 50\% WP & $600 \mathrm{~g} / \mathrm{ha}$ & \begin{tabular}{|c|}
3.29 \\
$(1.95)$ \\
\end{tabular} & $3.00(1.87)$ & \begin{tabular}{|c|}
2.87 \\
$(1.83)$ \\
\end{tabular} & $\begin{array}{c}2.43 \\
(1.71)\end{array}$ & \begin{tabular}{|c|}
1.99 \\
$(1.57)$
\end{tabular} & $\begin{array}{c}1.23 \\
(1.31)\end{array}$ & 2.30 & 84.17 \\
\hline 9. & T9: Untreated Check & - & $\begin{array}{l}12.87 \\
(3.64) \\
\end{array}$ & $\begin{array}{l}13.20 \\
(3.70)\end{array}$ & $\begin{array}{l}14.14 \\
(3.82)\end{array}$ & $\begin{array}{l}14.78 \\
(3.90)\end{array}$ & $\begin{array}{l}15.12 \\
(3.95) \\
\end{array}$ & $\begin{array}{l}15.41 \\
(3.98)\end{array}$ & 14.53 & - \\
\hline \multicolumn{3}{|c|}{ C.D. $(0.05 \%)$} & NS & 0.62 & 0.32 & 0.23 & 0.33 & 0.12 & \\
\hline & $\operatorname{Se}(m)$ & & - & 0.29 & 0.12 & 0.10 & 0.10 & 0.02 & & \\
\hline
\end{tabular}

Figure in brackets represents the square root transformed value * Mean of ten plants

It is evident from the given tables that the combination product of Lambda Cyhalothrin and Imidacloprid (Imidacloprid 6\% + Lambda Cyhalothrin 4\%SL) at three different doses viz; $500 \mathrm{ml} / \mathrm{ha}, 450 \mathrm{ml} / \mathrm{ha}$ and $400 \mathrm{ml} / \mathrm{ha}$ gave better outcome in respect with other tested insecticides. This may be due to the fact that Lambda Cyhalothrin is a broad spectrum contact insecticide whereas Imidacloprid is a systemic insecticide. Therefore when an insecticidal treatment is imposed with the above mentioned combination chemical Lambda Cyhalothrin and Imidacloprid, Lambda cyhalothrin initially reduces the insect population by quick knock down due to its contact nature and at the later days after treatment imposition Imidacloprid being systemic in nature maintains the pest population to very low tune. This explanation does not arise with other sole treatment impositions.

\section{Conclusion}

Thus, it can be summarized that the whitefly population build up was noticed during the fourth week of July with highest whitefly population at fourth week of September. The multivariate correlation studies suggest that maximum temperature is significantly positively correlated with the Whitefly population at $1 \%$ level of significance. Therefore the population of whitefly can be managed beforehand by application of Imidacloprid 6\% + Lambda Cyhalothrin 4\% SL (INOVEXIA) @ $450 \mathrm{ml} / \mathrm{ha}$ at fortnightly interval.

\section{Acknowledgement}

Authors are grateful to Dr. Hirak Chatterjee, Professor and Head, Department of Agricultural Entomology, Institute of Agriculture, PSB, Visva-Bharati, Santiniketan for extending 
necessary facilities and encouragement during course of investigation.

\section{References}

1. Abbas Q, Arif MJ, Gogi MD, Abbas SK, Karar H. Performance of imidacloprid, thiomethoxam, acetamaprid and a biocontrol agent (Chrysoperla carnea) against whitefly, jassid and thrips on different cotton cultivars. World Journal of Zoology. 2012; 7(2):141-146.

2. Ahmad M, Arif MI, Ahmad Z, Denholm I. Cotton whitefly (Bemisia tabaci) resistance to organophosphate and pyrethroid insecticides in Pakistan. Pest Management Science. 2002; 58(2):203-208.

3. Ashfaq S, Khan IA, Saeed M, Saljoqi AU, Manzoor F, Sohail $\mathrm{K}$ et al. Population dynamics of insect pests of cotton and their natural enemies. Sarhad Journal of Agriculture. 2011; 27(2):251-253.

4. Chaudhry MR, Guitchounts A. Cotton facts. Washington, DC, USA: International Cotton Advisory Committee, 2003, 35-83.

5. Chauhan R, Vekaria M, Chaudhary H, Chaudhary N. Seasonal incidence of sucking pests and their natural enemies in BT cotton. Journal of Entomology and Zoology Studies. 2017; 5(5):1274-1282.

6. Hargreaves H. List of recorded Cotton Insects of the World. List of recorded Cotton Insects of the World, 1948.

7. ICAR-All India Coordinated Research Project on Cotton - Annual Report. Central Institute of Cotton Research, Regional Station Coimbatore, 2015-16.

8. ICAR-All India Coordinated Research Project on Cotton - Annual Report. Central Institute of Cotton Research, Regional Station Coimbatore, 2017-18.

9. Kalkal D, Lal R, Dahiya KK, Singh M, Kumar A. Population dynamics of sucking insect pests of cotton and its correlation with abiotic factors. Indian Journal of Agricultural Research. 2015; 49(5):432-436.

10. Khan WS, Khan AG. Strategies for increasing cotton production. National seminar held at Agric. House, 21Agha Khan III Rd. Lahore, 1995, 26-27.

11. Kumar V, Shera PS, Aneja A. Seasonal Abundance of sucking insect pests on transgenic $\mathrm{Bt}$ cotton vis-à-vis weather parameters in Punjab, India. Acta Phytopathologica et Entomologica Hungarica. 2013; 48(1):63-74.

12. Mann RS, Schuster DJ, Cordero R, Toapanta M. Baseline toxicity of spiromesifen to biotype B of Bemisia tabaci in Florida. Florida Entomologist. 2012; 95(1):95-98.

13. Mayee CD, Singh $\mathrm{P}$, Dongre AB, Rao MRK, Raj S. Transgenic Bt. Cotton. CICR Technical Bulletin No: 22. Central Institute for Cotton Research Nagpur, 2011.

14. Muchhadiya DV, Saradava DA, Kabana BB. Population dynamics of insect pests and some of their natural enemies and their correlation with weather parameters on Bt cotton. Indian Journal of Agricultural Sciences. 2014; 84(5):572-578.

15. Naik VC, Kranthi S, Viswakarma R. Impact of newer pesticides and botanicals on sucking pest management in cotton under high density planting system (HDPS) in India. Journal of Entomology and Zoology Studies. 2017; 5(6):1083-1087.

16. Nelson MR, Nadeem A, Ahmed W, Orum TV. Global assessment of cotton virus disease. In Beltwide Cotton Conferences (USA), 1998, 161-162.
17. Shivanna BK, Nagaraja DN, Manjunatha M, Naik MI. Seasonal incidence of sucking pests on transgenic $\mathrm{Bt}$ cotton and correlation with weather factors. Karnataka Journal of Agricultural Sciences. 2009; 22(3):666-667.

18. Singh H. Status of insecticide resistance in Bemisia tabaci (Gennadius) on Bt cotton (Doctoral dissertation, Punjab Agricultural University, Ludhiana), 2017.

19. Singh S, Kumar A. Bio effectiveness of combination product of acetamiprid with cypermethrin, quinalphos and chlorpyriphos against insect pests of cotton. Indian Journal of Applied Entomology. 2006; 20:91-92. 